\title{
Balance of energetic electrons in zebra pattern solar radio sources
}

\author{
E.Ya.Zlotnik ${ }^{1}$, V.V.Zaitsev ${ }^{1}$, H.Aurass ${ }^{2}$ and G.Mann ${ }^{2}$ \\ ${ }^{1}$ IAP RAS, 46 Uljanov St., N. Novgorod 603950 Russia, email: zlot@appl.sci-nnov.ru \\ ${ }^{2}$ Astrophysicsl Institute Potsdam, Germany, email:haurass@aip.de
}

\begin{abstract}
An estimate of a typical life time of zebra pattern associated with escaping of fast particles from the trap into the loss-cone, is given. Besides, we estimate a number of fast electrons that is necessary to be injected into the trap in order to provide the observed brightness temperature of zebra stripes.
\end{abstract}

\section{Introduction}

An important problem of solar and stellar physics is the evaluation of productivity of acceleration mechanisms in different events arising due to super thermal particles. We consider type IV solar radio emission observed in a few minutes after the impulsive phase of a flare. Its dynamic spectrum is a broadband continuum with high brightness temperature, and it often shows different kinds of fine structure (FS) the most prominent of which are broadband quasi-periodic pulsations (BBP) and zebra-pattern (ZP). An example of such FS was recorded by Tremsdorf Observatory (AIP) on October 25, 1994 and analyzed in detail in Aurass et al. (2003) and Zlotnik et al. (2003). The BBP are shown to result from periodically repeated injections of fast electrons into the asymmetric magnetic trap and the stream instability of plasma waves (similar to type III bursts). The $\mathrm{ZP}$ is due to a plasma wave instability at the levels of double plasma resonance (DPR) in magnetic loop. BBP resulting from the periodic injection of electrons into the trap are considered as a specific kind of pumping up the source of zebra-pattern. An estimate of a typical life time of ZP associated with escaping of fast particles from the trap into the loss-cone, is given. Also, we estimate a number of fast electrons that is necessary to be injected into the trap in order to provide the observed brightness temperature of zebra stripes.

\section{Equation of electron balance in the trap}

Fast electrons are accumulated in the loop due to the source, arising from some acceleration mechanism, and precipitate into the loss cone due to the quasi-linear diffusion of the electron distribution function because of interaction with plasma waves. Under some assumptions the balance of the hot electrons $n_{h}$ in the loop (trap) is described by the following equation:

$$
\frac{d n_{h}}{d t} \approx-\frac{n_{h}}{\tau}+\frac{3 I}{2 S_{1} \sigma_{1} L}
$$

where

$$
\tau=\frac{8}{3} \frac{\sigma_{1} L}{V}, \frac{S_{1}}{S_{1}+S_{2}}
$$

$S_{1}$ and $S_{2}$ are cross-sections of the loop footpoints, $V$ is the velocity of injected hot 
electrons, $\sigma_{1}=B_{1} / B_{\text {top }}$ is the mirror ratio, $L$ is the trap length, $I\left(\mathrm{~s}^{-1}\right)$ is the rate of injection of the total number of fast electrons into the trap.

\section{The time of zebra pattern decay}

After switching the acceleration mechanism off in $(2.1)(I=0)$ the density of fast electrons in the trap exponentially decreases:

$$
n_{h}(t)=\frac{4 I}{V\left(S_{1}+S_{2}\right)} \exp (-t / \tau) .
$$

So, the time (2.2) determines the time of fast particle precipitation and decay of nonequilibrium distribution function of electrons responsible for $\mathrm{ZP}$ origin. Using parameters of the trap given in Zlotnik et al. (2003), we obtain the estimation of the precipitation time as $\tau \sim(40 \div 50)$ s, that coincides with the time of ZP decay after stopping BBP.

\section{Power of the fast particle source $I$}

The value $I$ may be estimated with respect to the observed brightness temperature of $\mathrm{ZP}\left(T_{b} \approx 1.4 \cdot 10^{10} \mathrm{~K}\right)$ which is determined by plasma wave energy density $W_{L}$ (Zaitsev \& Stepanov (1983)). In the steady-state regime of loss cone instability when escaping of electrons into the loss cone is compensated by a source, the value $W_{L}$ may be expressed via parameters of the trap, the electron velocity and the source power $I$ (see Shaposhnikov (1988) more in detail). All this allows to estimate the productivity as $I \sim 2.5 \times 10^{32} \mathrm{~s}^{-1}$ for our event (see Zaitsev et al. (2004)). This estimation seems to be quite reasonable one because it is in 3-4 orders of magnitude lower than the efficiency of electrons acceleration during solar flares. The estimate of parameter $I$ can be compared with observations if spatially and spectrally resolved data are available.

\section{Conclusions}

Basing on the observations of ZP event on October 25, 1994 and the theory suggested in Aurass et al. (2003) and Zlotnik et al. (2003) we have estimated a typical life time of zebra pattern defined by escaping of fast particles from the trap into the loss-cone. It is well conformed with observed life time of $\mathrm{ZP}$ after switching the source off. Besides we have got a number of fast electrons that is necessary to be injected into the trap in order to provide the observed brightness temperature of zebra stripes. The necessary productivity of fast electrons appears to be in three orders of magnitude less than the rate of electron acceleration during a medium power solar flare that proves the reasonability of the estimations obtained.

\section{References}

Aurass H., Klein K.-L., Zlotnik E.Ya., Zaitsev V.V. 2003 A\&A 410, 1001-1010.

Zlotnik E.Ya., Zaitsev V.V., Aurass H., Mann G., Hofman A. 2003 A\&A 410, 1011-1022.

Zaitsev, V.V., Stepanov, A.V. 1983 Solar Phys. 88, 297-303.

Shaposhnikov, V.E. 1988 Sov.Astron.Lett. 14, 275-277.

Zaitsev, V.V., Zlotnik, E.Ya., Aurass, H. 2005, Astronomy Letters, V. 31, No. 3. 